\title{
Anderson localization and correlated disorder
}

\author{
F.M.Izrailev \\ Instituto de Física, BUAP \\ Puebla, México
}




\title{
Anderson localization and correlated disorder
}

\author{
F.M.Izrailev \\ Instituto de Física, BUAP \\ Puebla, México
}




\section{Outlook}

- Anderson localization: White-noise disorder

- Kronig-Penney and Anderson models

- Localization length

- Correlated disorder

- Experiments with single-mode waveguides

- Bi-layered structures 


\section{1-D Schrödinger equation :}

$$
-\frac{\hbar^{2}}{2 m} \psi^{\prime \prime}(x)+U(x) \psi(x)=E \psi(x)
$$

\section{Classical wave equation :}

$\psi^{\prime \prime}(x)+k^{2} \psi(x)=U(x) \psi(x) \quad$ with $\quad E=k^{2}$

Main interest is in the structure of eigenstates and transport properties for a disorder with long-range correlations in $U(x)$ 


\section{Continuous models with white-noise potentials}

with (weak) random potential, $\langle\boldsymbol{U}(\boldsymbol{x})\rangle=0 ; \quad\left\langle\boldsymbol{U}^{2}(\boldsymbol{x})\right\rangle=\sigma^{2}<<1$

The problem is solved exactly:

$$
\begin{aligned}
\langle\boldsymbol{T}\rangle & =\sqrt{\frac{2}{\pi}}\left(\frac{\boldsymbol{l}_{\infty}}{\boldsymbol{L}}\right)^{3 / 2} \exp \left(-\frac{\boldsymbol{L}}{2 \boldsymbol{l}_{\infty}}\right) \int_{0}^{\infty} \frac{\boldsymbol{z}^{2}}{\boldsymbol{c h} \boldsymbol{e x p}\left(-\boldsymbol{z}^{2} \frac{\boldsymbol{l}_{\infty}}{2 \boldsymbol{L}}\right) d z} \\
\left\langle\boldsymbol{T}^{2}\right\rangle & =\sqrt{\frac{2}{\pi}}\left(\frac{\boldsymbol{l}_{\infty}}{\boldsymbol{L}}\right)^{3 / 2} \exp \left(-\frac{\boldsymbol{L}}{2 \boldsymbol{l}_{\infty}}\right) \int_{0}^{\infty} \frac{\left(2 \boldsymbol{z}^{2}+\boldsymbol{z} \operatorname{sh} 2 \boldsymbol{z}\right)}{4 \boldsymbol{c h}^{3} z} \exp \left(-z^{2} \frac{\boldsymbol{l}_{\infty}}{2 \boldsymbol{L}}\right) d z
\end{aligned}
$$

Single-parameter scaling: All transport properties depend on one parameter only $\quad \xi=l_{\infty} / L$ 


\section{Anderson localization}

in completely random potentials all eigenstates are exponentially

$$
|\psi(x)| \sim \exp \left(-\frac{\left|x-x_{0}\right|}{l_{\infty}(E)}\right)
$$

localized

$l_{\infty}(E)$ - localization length (in infinite $\boldsymbol{x}$-space)

$$
\langle\ln T\rangle=-2 L / l_{\infty} \quad ! ! !
$$

For finite samples of size $L$ the transmission coefficient $T$ is

(a) strongly localized states (insulator)

$$
\langle T\rangle \sim \exp \left(-2 L / l_{\infty}\right) \quad l_{\infty}<L L
$$

(b) weakly localized states (conductor)

$$
\langle\boldsymbol{T}\rangle \approx 1-2 \boldsymbol{L} / \boldsymbol{l}_{\infty} \quad \boldsymbol{l}_{\infty}>\boldsymbol{L}
$$




\section{Anderson tight-binding model}

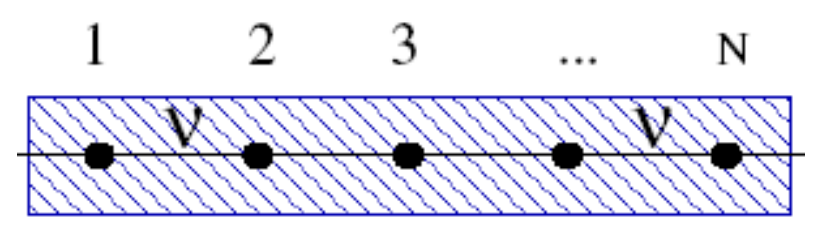

$\varepsilon_{n}$ - site energies of an electron

$v=1$ - hopping matrix element

$$
\nu \psi_{n+1}+\nu \psi_{n-1}=\left(E+\varepsilon_{n}\right) \psi_{n}
$$

Localization length - White-noise disorder

$$
\lambda=\boldsymbol{l}_{\infty}^{-1}=\frac{\sigma^{2}}{8 \sin ^{2} \mu} \quad \begin{gathered}
\sigma^{2}=\left\langle\varepsilon_{n}^{2}\right\rangle \\
\mathbf{2} \cos \boldsymbol{\mu}=\boldsymbol{E}
\end{gathered}
$$




\section{Kronig-Penney models}

(a) amplitude disorder --

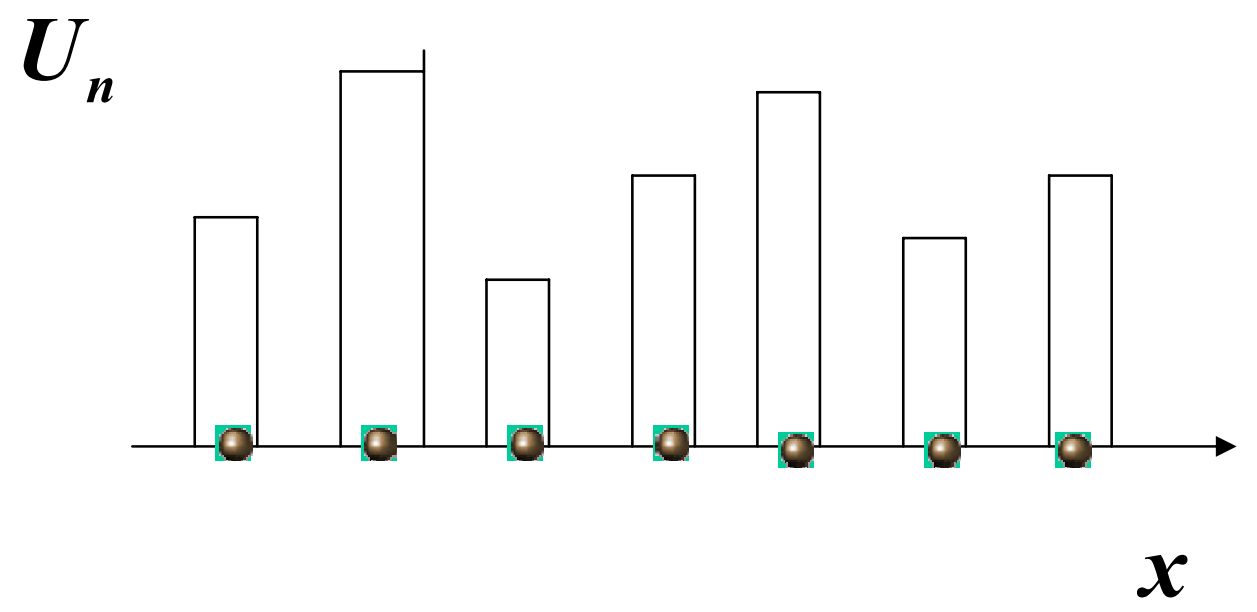

(b) positional disorder --

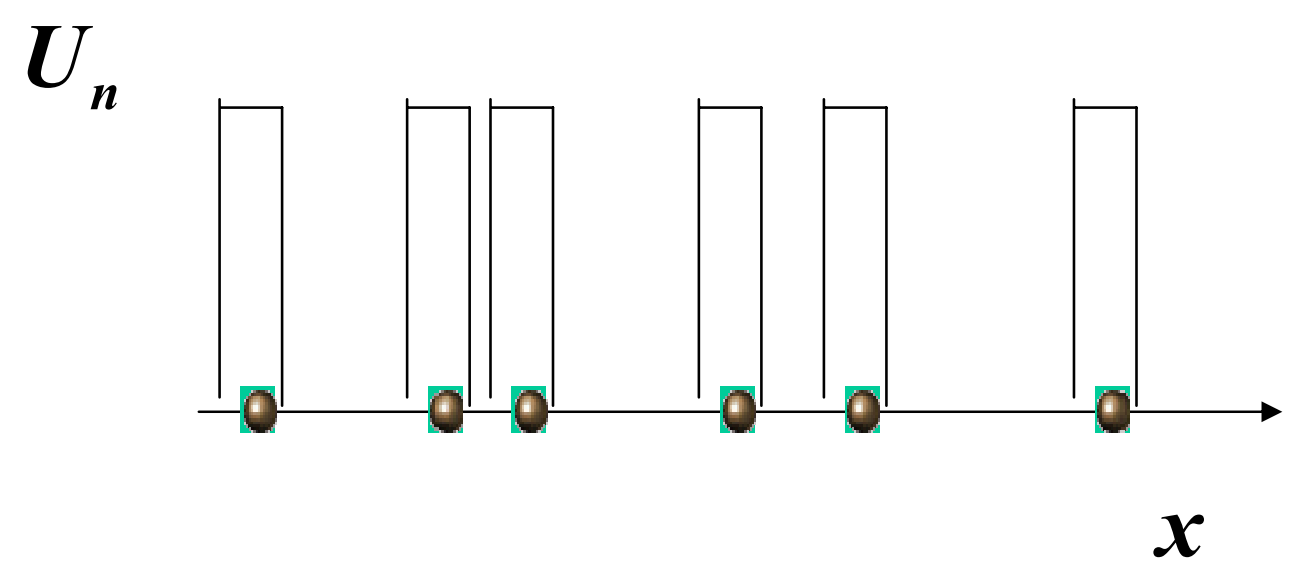




\section{Kronig-Penney model}

$$
\psi^{\prime \prime}(\boldsymbol{x})+\boldsymbol{k}^{2} \psi(\boldsymbol{x})=\sum_{n=-\infty}^{\infty} \boldsymbol{U}_{n} \psi\left(\boldsymbol{x}_{n}\right) \delta\left(\boldsymbol{x}-\boldsymbol{x}_{n}\right)
$$

Here $U_{n}$-- amplitude of the n-th delta-scatterer at $x=x_{n}$

Experimental setup, two limit cases:

(a) amplitude disorder -- $\boldsymbol{x}_{\boldsymbol{n}}=\boldsymbol{d} \boldsymbol{n} ; \quad \boldsymbol{U}_{n}$ - random

(b) positional disorder -- $\boldsymbol{x}_{\boldsymbol{n}}=\boldsymbol{d} \boldsymbol{n}+\xi_{n} ; \xi_{n} \quad$ - random

$$
U_{n}=\boldsymbol{U}=\text { const }
$$




\section{Kronig-Penney model: Amplitude disorder}

$$
\psi_{n+1}+\psi_{n-1}=\left(2 \cos \mu+\frac{U_{n}}{k} \sin \mu\right) \psi_{n}
$$

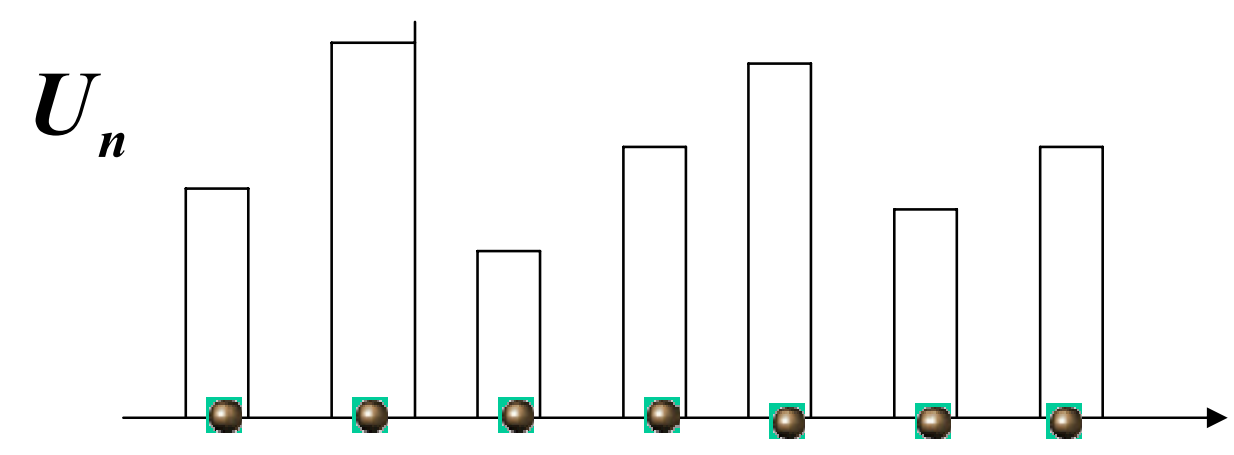

$\mu=\boldsymbol{k d}$

$\boldsymbol{x}$

where $d$ is spacing between scatterers

$$
\boldsymbol{U}_{n}=\overline{\boldsymbol{U}}+\xi_{n} \quad \text { and } \quad \overline{\boldsymbol{U}}=<\boldsymbol{U}_{n}>
$$




\section{Kronig-Penney model: Localization length}

$$
\begin{array}{cc}
\lambda=\boldsymbol{l}_{\infty}^{-1}=\frac{\sigma^{2}}{8 \boldsymbol{k}^{2}} \frac{\sin ^{2} \mu}{\sin ^{2} \gamma} & \boldsymbol{E}=\boldsymbol{k}^{2} \quad \boldsymbol{k}=\omega / \boldsymbol{c} \\
2 \cos \mu+\frac{\bar{U}}{\boldsymbol{k}} \sin \mu=2 \cos \gamma & \sigma^{2}=\left\langle\xi_{n}^{2}\right\rangle<<\overline{\boldsymbol{U}}^{2} \\
\mu=\boldsymbol{k d}
\end{array}
$$

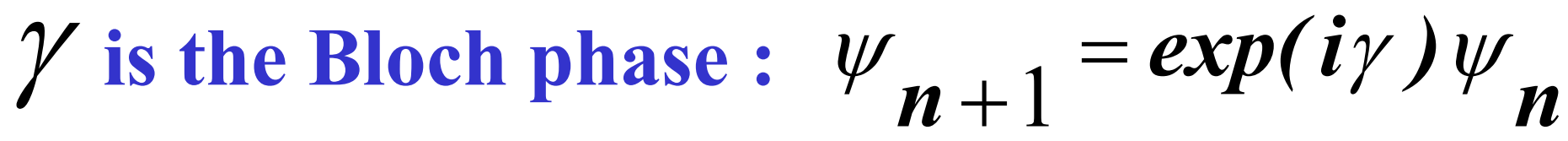

$$
\lambda \text { - Lyapunov exponent }
$$

For single-mode waveguide: $k=\sqrt{(\omega / c)^{2}-(\pi / d)^{2}}$ 


\section{Localization length - correlated disorder}

$$
\lambda=l_{\infty}^{-1}=\frac{\sigma^{2}}{8 \boldsymbol{k}^{2}} \frac{\sin ^{2} \mu}{\sin ^{2} \gamma} W(2 \mu)
$$

where $W(2 \mu)=1+2 \sum_{m=1}^{\infty} K(m) \cos (2 \mu m)$

and $K(m)=<\varepsilon_{n} \varepsilon_{n+m}>/<\varepsilon_{n}^{2}>$ is the pair correlator

$$
K(\boldsymbol{m})=\frac{1}{\pi} \int_{0}^{\pi} W(\mu) \cos (2 \mu m) d \mu
$$




\section{How to construct the potential from}

a given pair correlator?

Method: $\quad \varepsilon_{n}=\sigma \sum_{m=-\infty}^{\infty} \beta_{m} Z_{n+m}$

where $\quad \beta_{m}=\frac{1}{\pi} \int_{0}^{\pi} \sqrt{W(\mu)} \cos (2 \mu m) d \mu$

$Z_{n} \quad$ are random numbers with the zero mean and unitary variance 


\section{Numerical simulation - Anderson model}

$$
\begin{gathered}
\Lambda_{0}=l_{\infty}^{-1} /\left(l_{\infty}^{-1}\right)_{w} \\
\left(l_{\infty}^{-1}\right)_{w}=\frac{\sigma^{2}}{8 \sin ^{2} \mu} \\
2 \cos \mu=E
\end{gathered}
$$

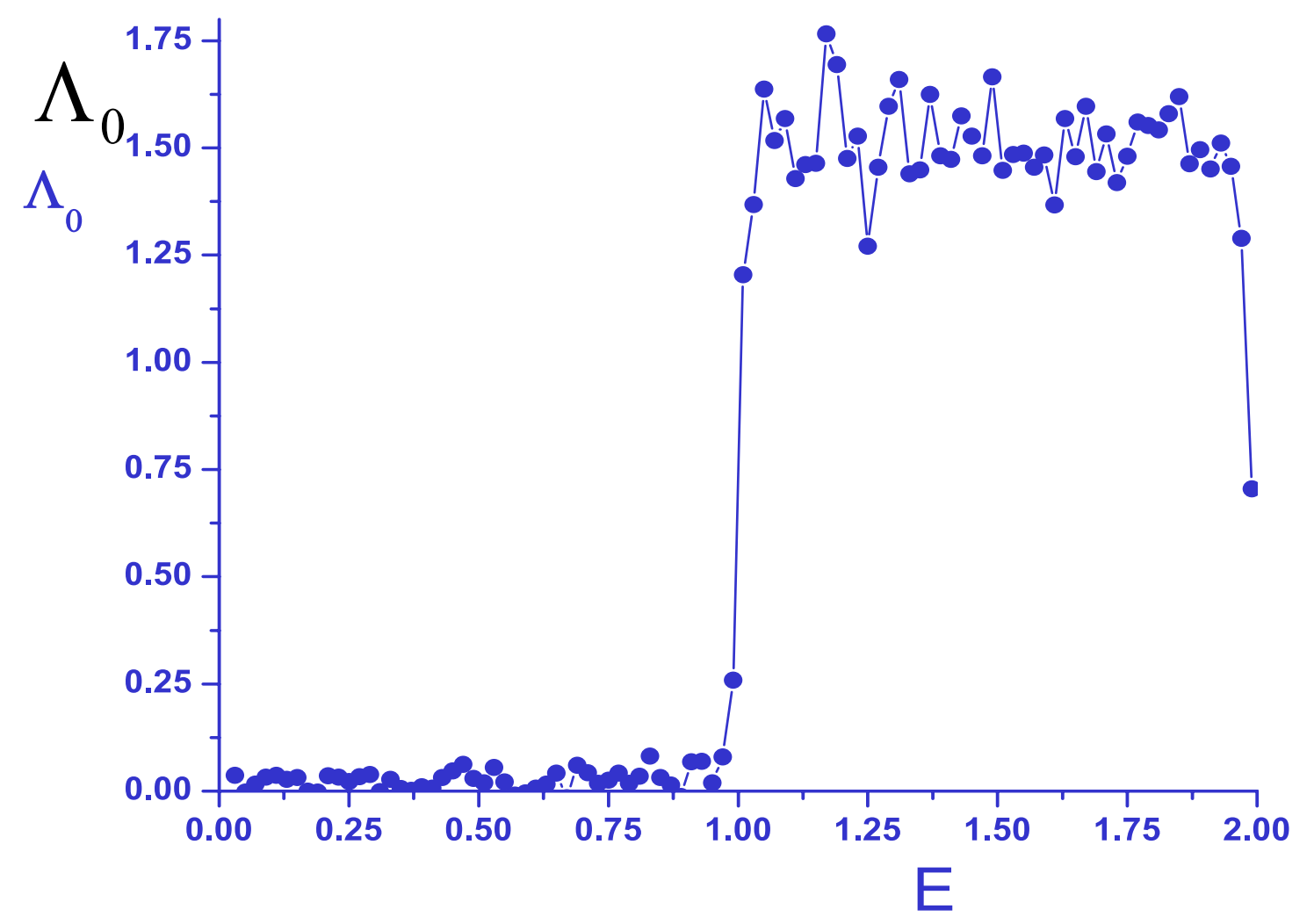




\section{Numerical confirmation: Kronig-Penney model}
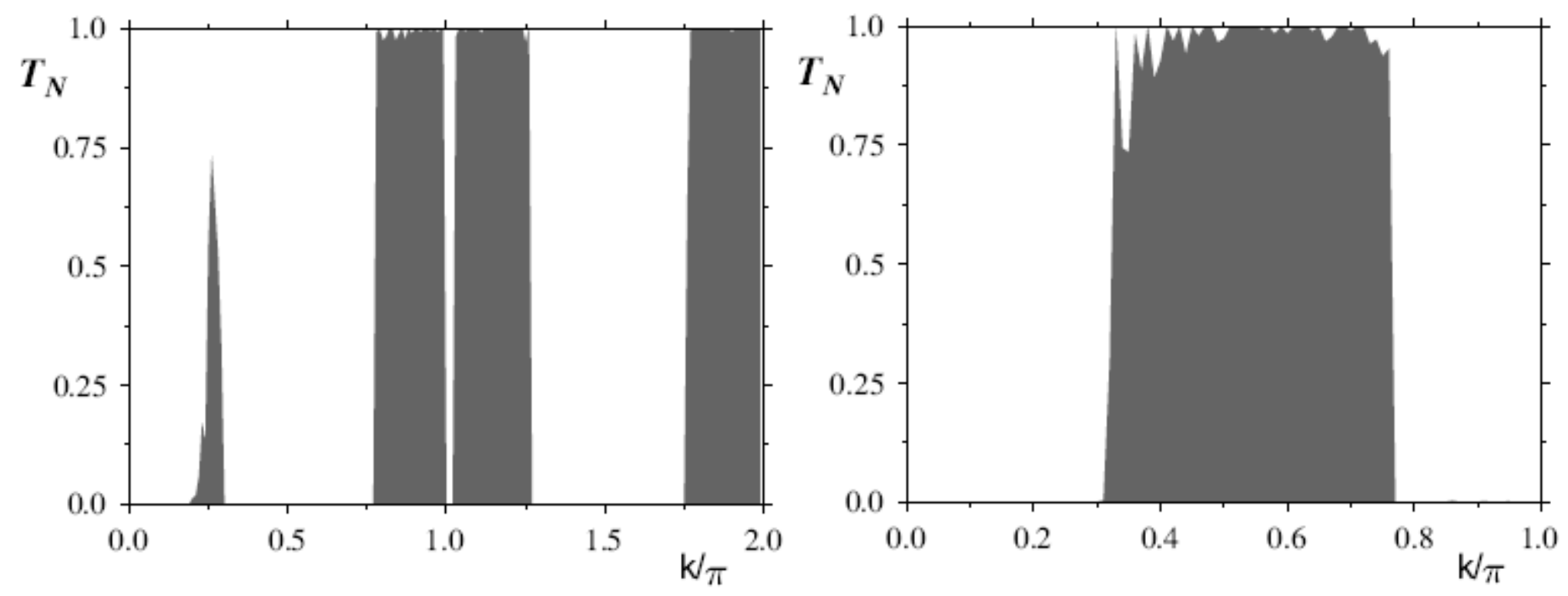

Figure 17: Transmission coefficient vs. energy for data of Fig.16. (a): correlations in the potential correspond to the dependence $W(\mu)=0$ outside the interval $[\pi / 4,3 \pi / 4]$ in the first zone; two zones are shown, $0<$ $k / \pi<2$, see Fig.16a. (b): the same for the complementary dependence $W(\mu)$ in the first zone only, see Fig.16b. The length of sequence $U_{n}$ in both cases is $N=1000$ (after [KI00]). 


\section{Single-mode waveguide: Experimental setup}

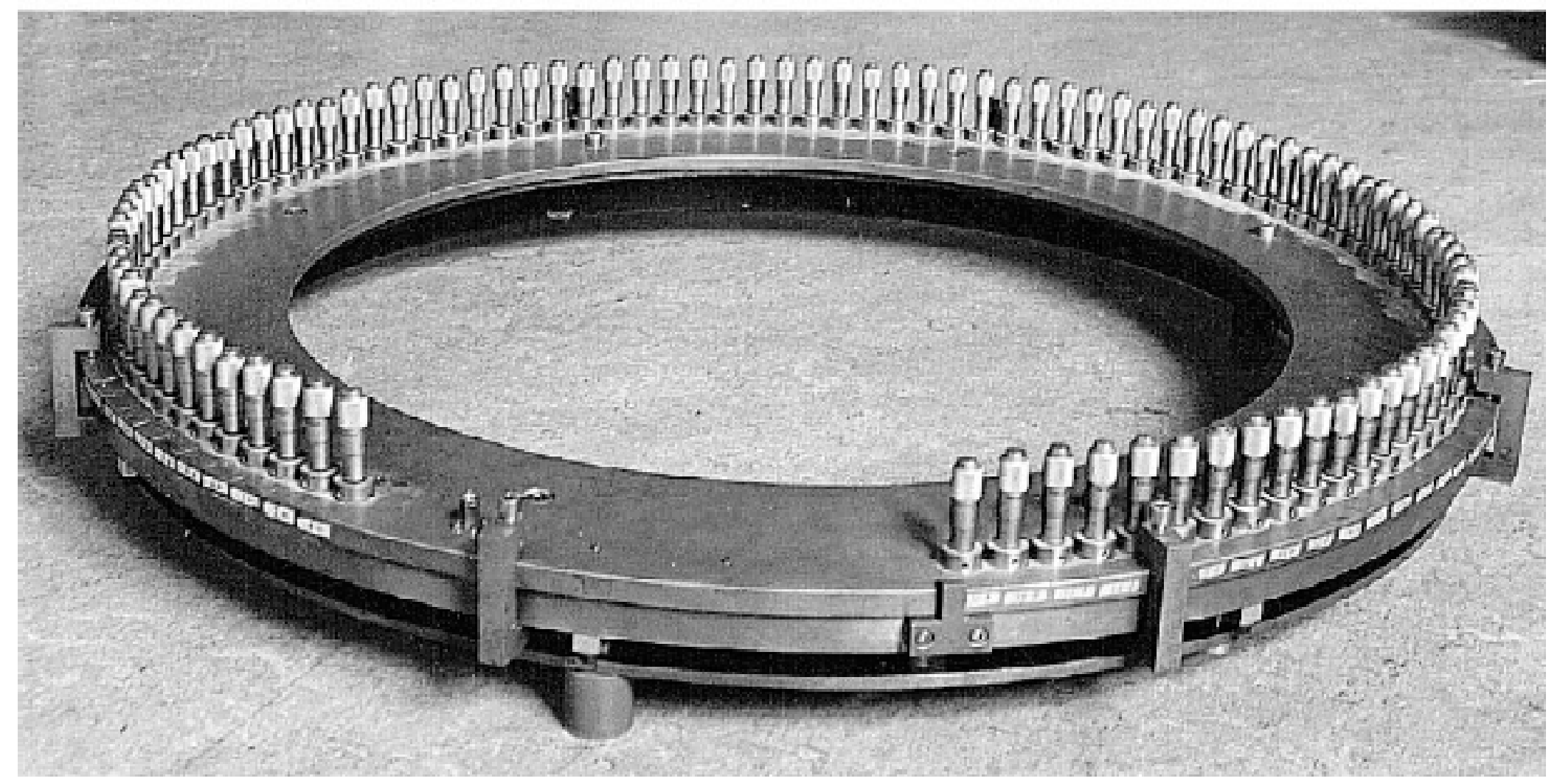

U.Kuhl, F.Izrailev, A.Krokhin, H.-J.Stöckmann, Appl. Phys. Lett., 77 (2000) 633. 


\section{Single-mode waveguide: Experimental setup}

634 Appl. Phys. Lett., Vol. 77, No. 5, 31 July 2000
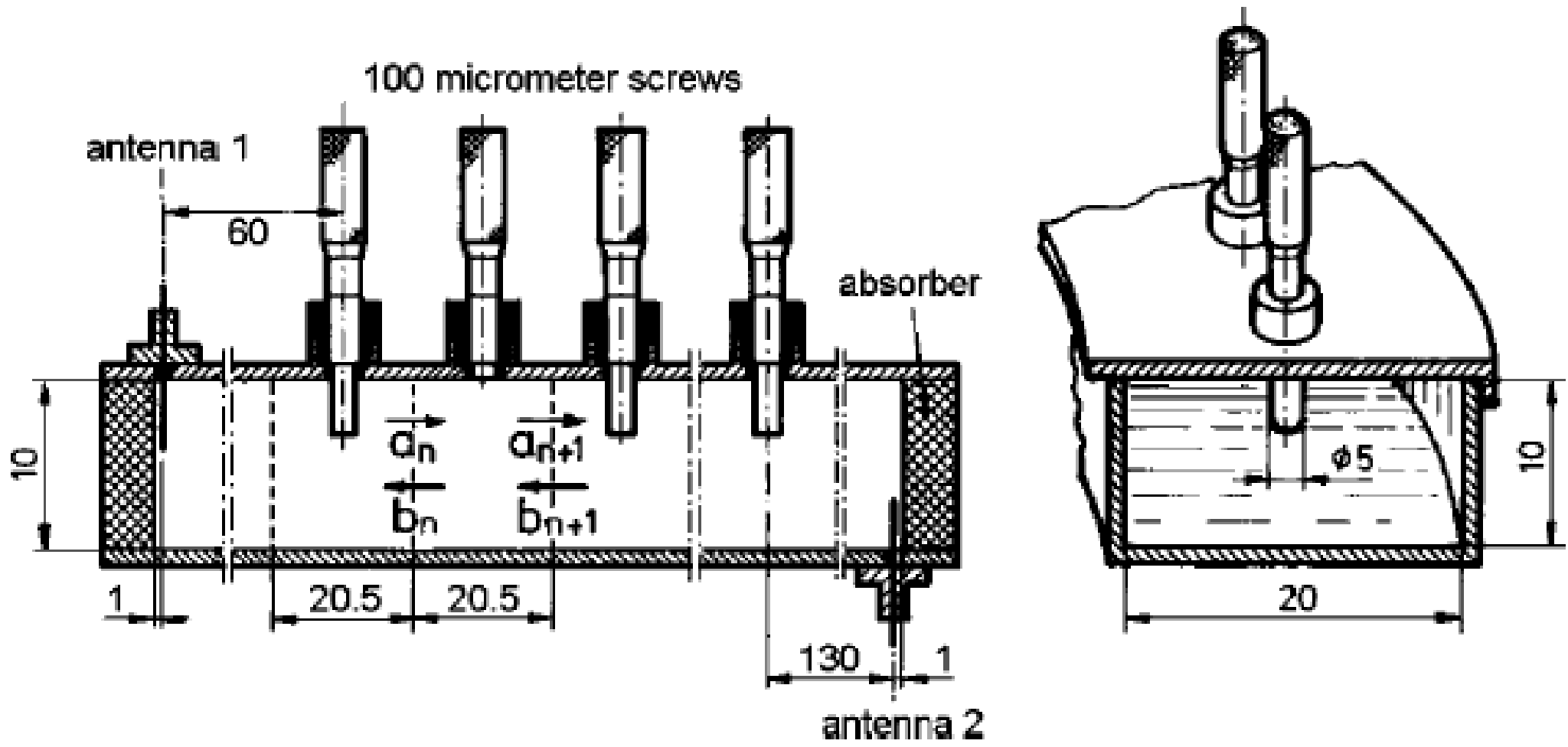

$$
v=7.5 \div 15.0 \mathrm{GHz}
$$




\section{Transmission coefficient for 2 correlated sequences}

Appl. Phys. Lett., Vol. 77, No. 5, 31 July 2000

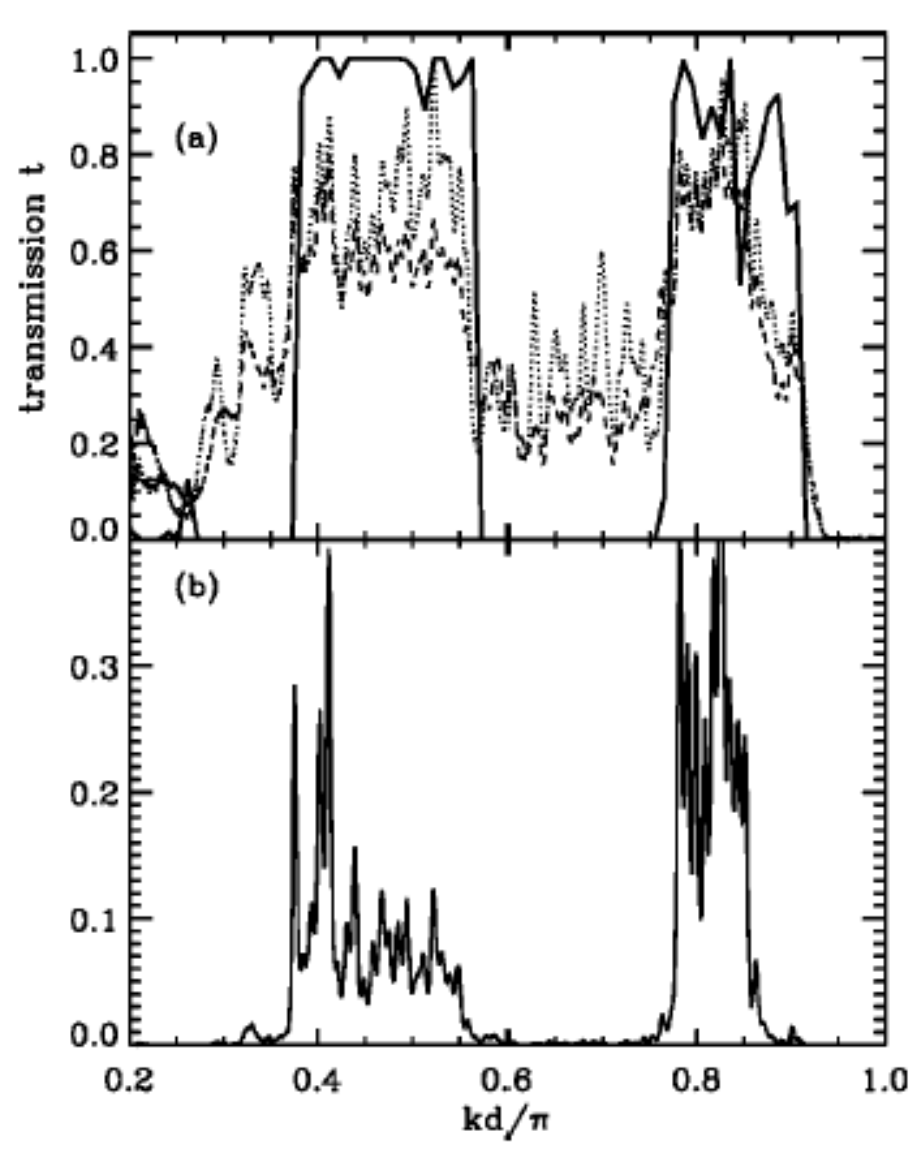

$1^{\text {st }}$ sequence
Kuhl et al.

635

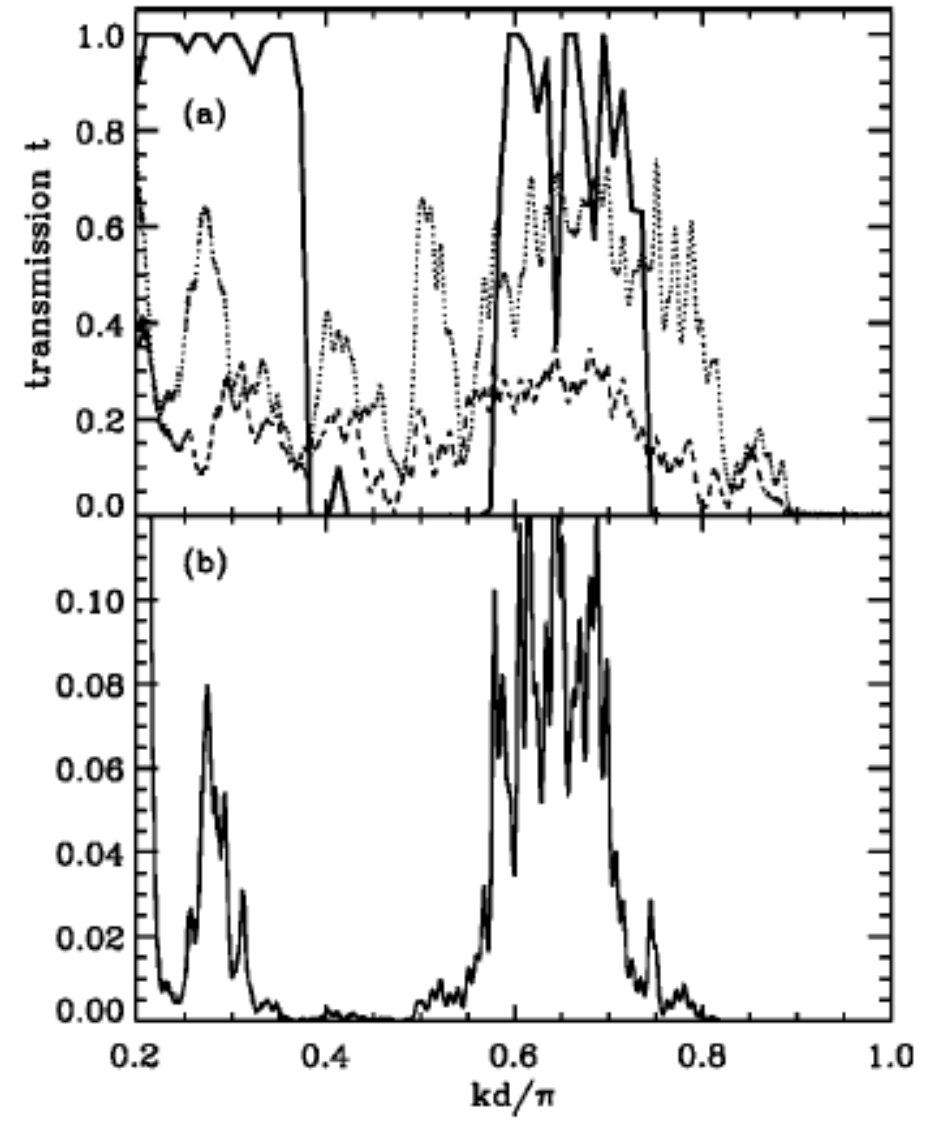

N

100

500 


\section{Selective reflection - enhancement of localization}

\section{Example}

$2 \cos \mu=E \quad 0<\mu<\pi$

$$
\lambda=l_{\infty}^{-1}=\frac{\sigma^{2}}{8 \boldsymbol{k}^{2}} \frac{\sin ^{2} \mu}{\sin ^{2} \gamma} W(2 \mu)
$$

The function $W(\mu)$ is normalized :

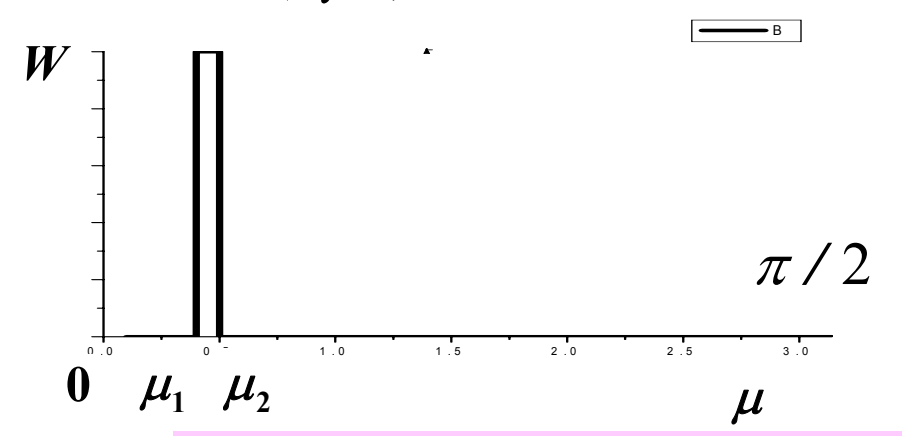

$$
\int_{0}^{\pi} W(\mu) d \mu=1
$$

$$
\Delta=E_{2}-E_{1}
$$

For this case, $K(m)=\frac{c^{2}}{\pi m}\left[\sin \left(2 m \mu_{2}\right)-\sin \left(2 m \mu_{1}\right)\right]$

$$
\text { and } \quad l_{\infty}^{-1}=\frac{\pi \sigma^{2}}{16 \sin ^{2} \mu\left[a \cos \left(E_{2} / 2\right)-a \cos \left(E_{1} / 2\right)\right]} \approx \frac{\pi \sigma^{2}}{16 \Delta \sin ^{2} \mu}
$$




\section{Enhancement of localization - experiment}
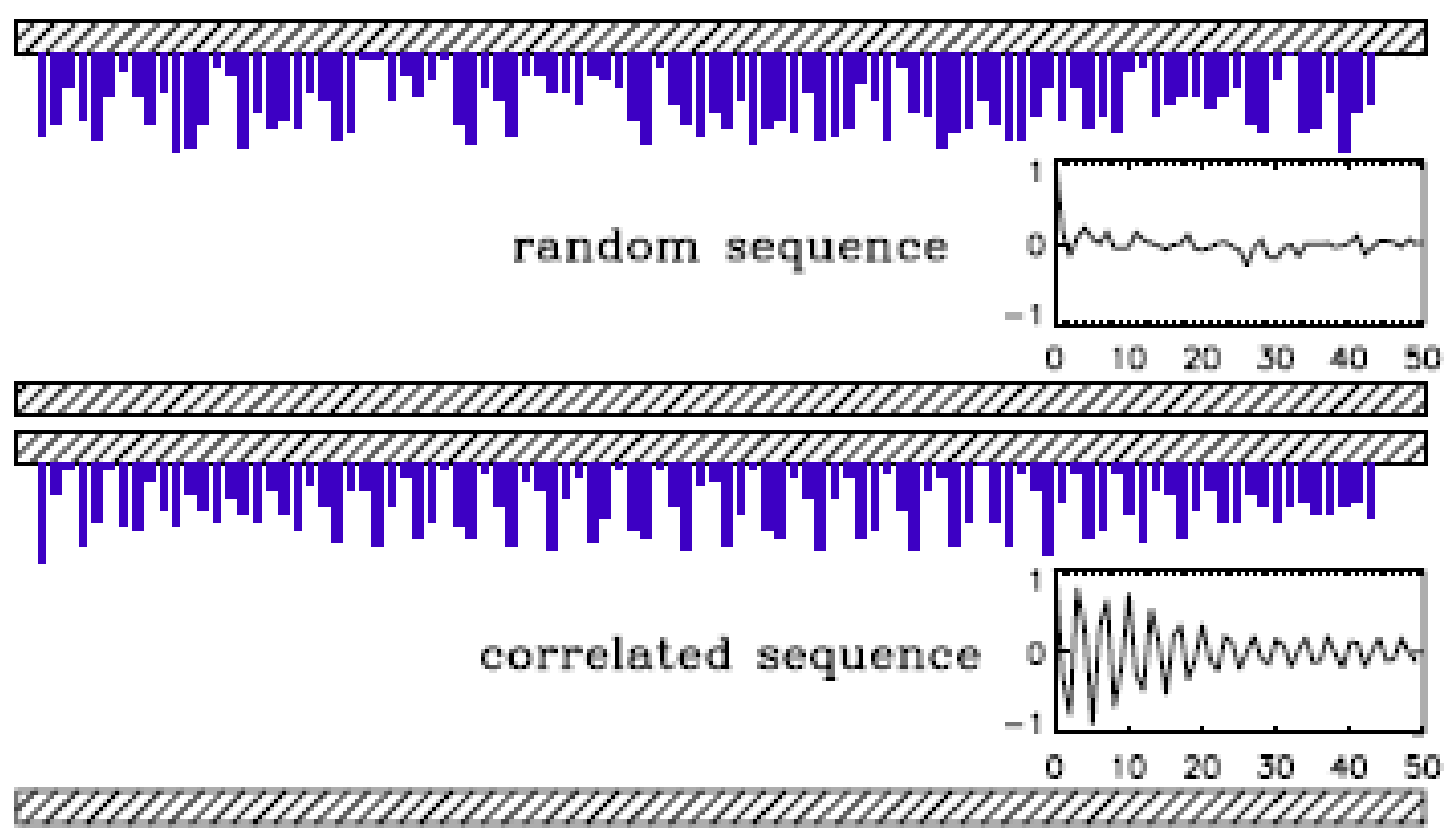

FIG. 2 (color online). Profile of intrusion of all 100 micrometer screws into the waveguide for uncorrelated and correlated random sequence $U_{n}$. The insets show the corresponding correlation function calculated from the micrometer screw depths [Eq. (7)].

U.Kuhl, F.M.Izrailev, A.A.Krokhin, Phys. Rev. Lett., 100 (2008) 126402. 


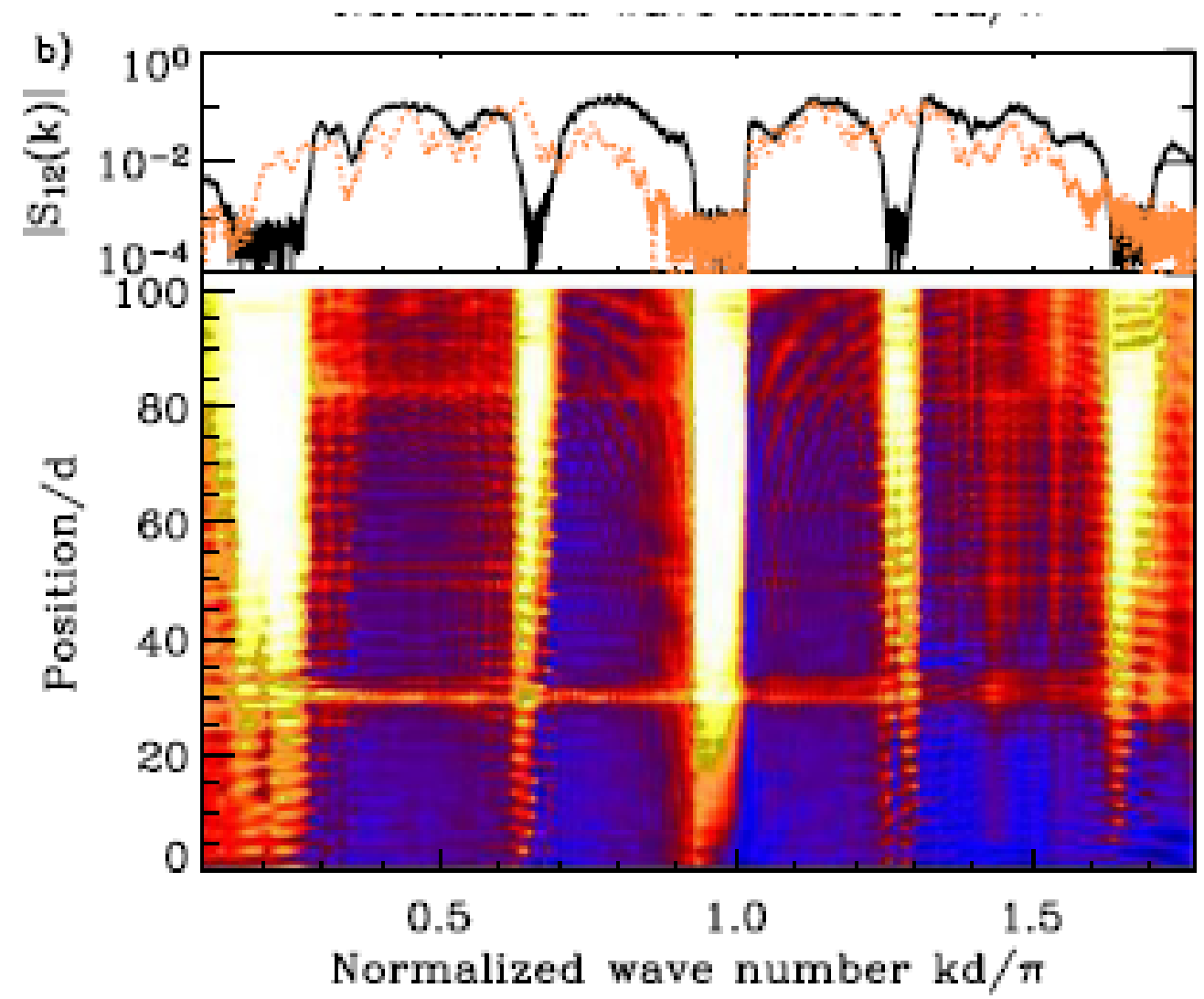

FIG. 3 (color online). Transmission spectrum $\left|S_{12}(k)\right|$ versus position $n$ (vertical scale) of the moving antenna is shown for uncorrelated (a) and correlated (b) disorder. The transmission spectrum $\left|S_{12}\right|$ through the whole waveguide is shown by black solid line at the top of each panel. For comparison, the transmission through uncorrelated disorder is added on the top of panel (b) by a yellow dotted line. 


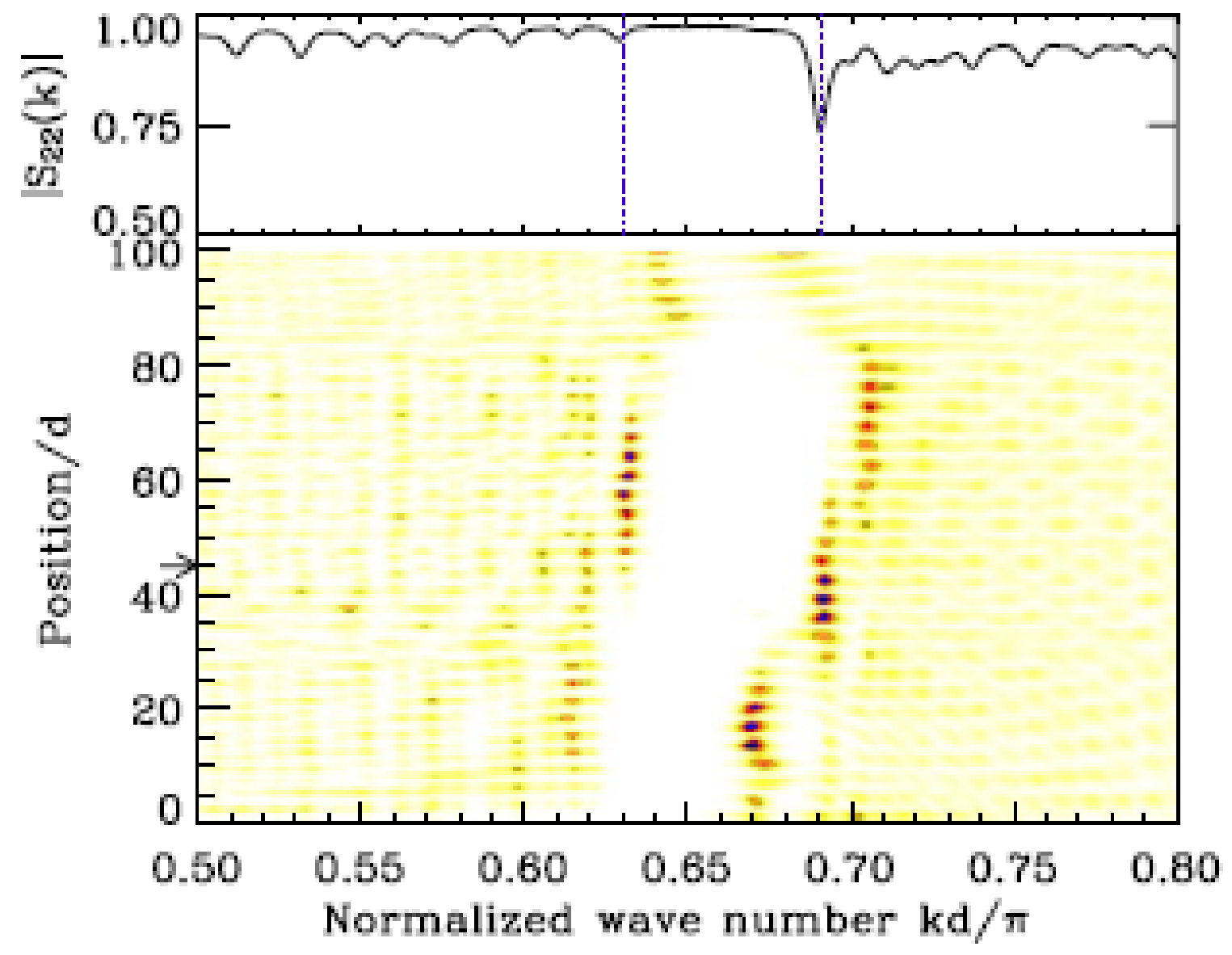

FIG. 5 (color online). Details of Fig. 4 for wave numbers between 0.50 and 0.80 . In this range of wave numbers one can see several enhanced-localized states. Sharp drop of $\left|S_{22}\right|$ at $k d / \pi \approx 0.691$ is due to localized states centered at $n \approx 40$. 


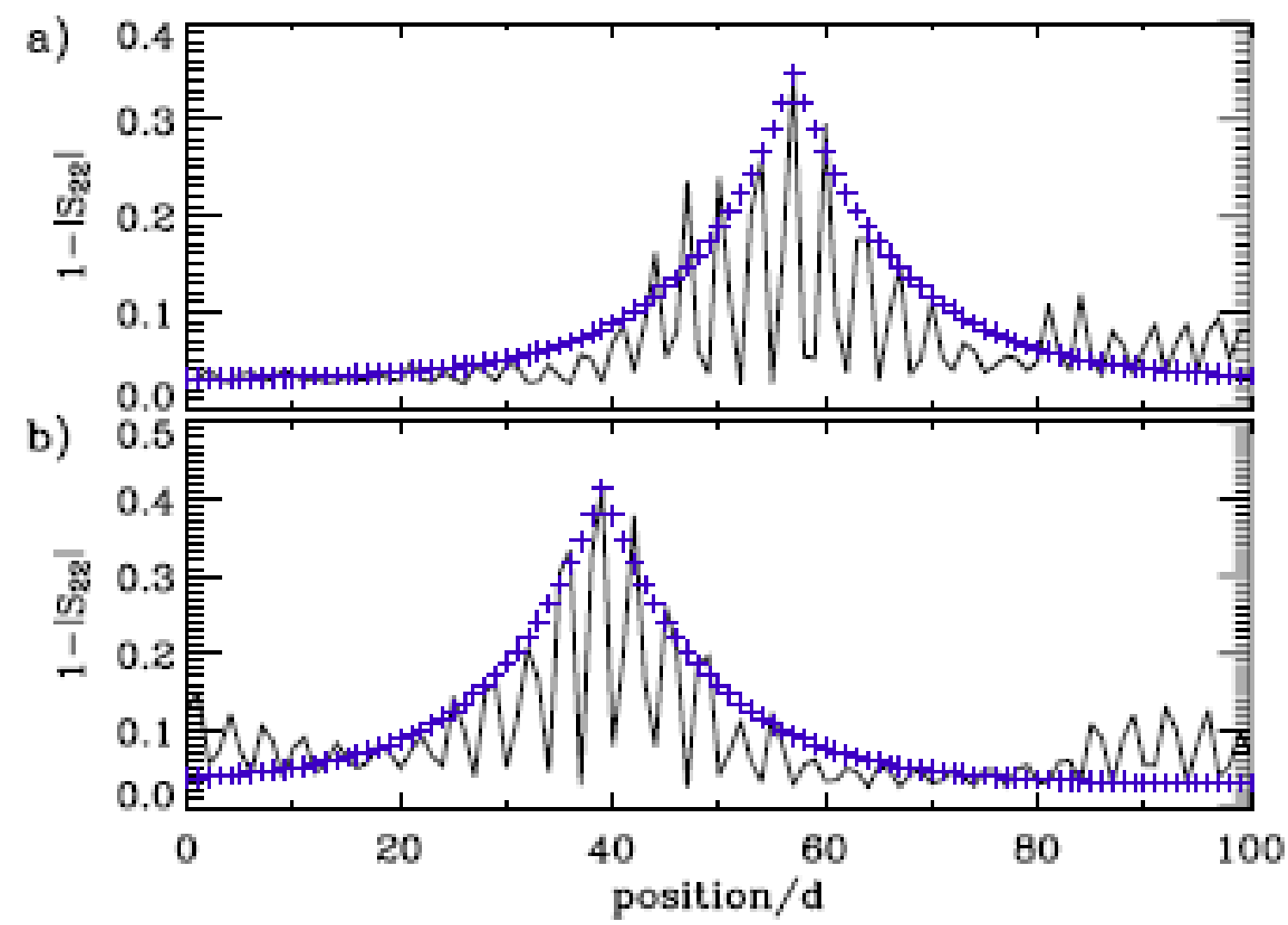

FIG. 6 (color online). Profiles of two enhanced-localized states at $k d / \pi \approx 0.63$ (a) and 0.69 (b) for the correlated sequence (marked by vertical dotted lines in Figs. 4 and 5. Graph (b) is the state responsible for a sharp decrease of $\left|S_{22}\right|$ shown at the top of Fig. 5. Crosses show an exponential decay with a localization length of 10 scatterer spacings. 


\title{
Bi-layered structures
}

PRL 102, 203901 (2009)

PHYSICAL REVIEW LETTERS

\section{Localization in Correlated Bilayer Structures: From Photonic Crystals to Metamaterials and Semiconductor Superlattices}

\author{
F. M. Izrailev ${ }^{1}$ and N. M. Makarov ${ }^{2}$
}

In a unified approach, we study the transport properties of periodic-on-average bilayered photonic crystals, metamaterials, and semiconductor superlattices. Our consideration is based on the analytical expression for the localization length derived for the case of weakly fluctuating widths of layers and takes into account possible correlations in disorder. We analyze how the correlations lead to anomalous properties of transport. In particular, we show that for quarter stack layered media specific correlations can result in a $\omega^{2}$ dependence of the Lyapunov exponent in all spectral bands.

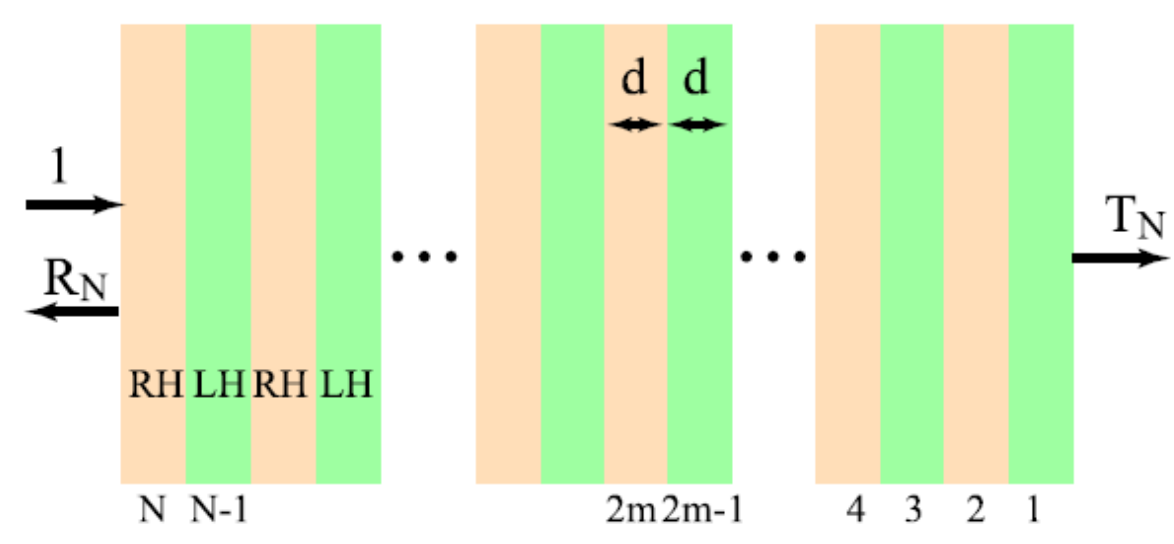




\section{The model}

In what follows we consider a propagation of monochromatic electromagnetic TE-wave of frequency $\omega$ through an infinite array (stack) of two alternating layers (slabs). Every kind of layers is respectively specified by permittivity (dielectric constant) $\varepsilon_{a, b}$, magnetic permeability $\mu_{a, b}$, corresponding refractive index $n_{a, b}$, impedance $Z_{a, b}$ and wave number $k_{a, b}$,

$$
\begin{array}{lll}
n_{a}=\sqrt{\varepsilon_{a}} \sqrt{\mu_{a}}, & k_{a}=\omega n_{a} / c=\omega \sqrt{\varepsilon_{a}} \sqrt{\mu_{a}} / c, & Z_{a}=\sqrt{\mu_{a} / \varepsilon_{a}} ; \\
n_{b}=\sqrt{\varepsilon_{b}} \sqrt{\mu_{b}}, & k_{b}=\omega n_{b} / c=\omega \sqrt{\varepsilon_{b}} \sqrt{\mu_{b}} / c, & Z_{b}=\sqrt{\mu_{b} / \varepsilon_{b}} .
\end{array}
$$

Within every $a$ or $b$ layer, the electric field of the wave obeys the wave equation with two boundary conditions formulated on the interfaces between slabs,

$$
\begin{aligned}
& \left(\frac{d^{2}}{d z^{2}}+k_{a, b}^{2}\right) \psi_{a, b}(z)=0, \\
& \psi_{a}\left(z_{i}\right)=\psi_{b}\left(z_{i}\right), \quad \mu_{a}^{-1} \psi_{a}^{\prime}\left(z_{i}\right)=\mu_{b}^{-1} \psi_{b}^{\prime}\left(z_{i}\right) .
\end{aligned}
$$

Here $z$-axis is stretched along the array of bi-layers perpendicular to the stratification, $z_{i}$ stands for the coordinate of an interface. 


\section{Random widths of slabs with correlations}

A disorder is incorporated in the structure via the random widths of the slabs (the positional disorder),

$$
\widetilde{a}(n)=a+\varrho_{a}(n), \quad \widetilde{b}(n)=b+\varrho_{b}(n) .
$$

Here $n$ enumerates the elementary $a b$-cells, $a$ and $b$ are the average widths of layers and $\varrho_{a}(n), \varrho_{b}(n)$ stand for small variations of the widths. In the absence of disorder the array of bi-layers is periodic with the period $d=a+b$. The random sequences $\varrho_{a}(n), \varrho_{b}(n)$ are supposed to be statistically homogeneous with zero average, $\left\langle\varrho_{a}(n)\right\rangle=\left\langle\varrho_{b}(n)\right\rangle=0$, and binary (pair) correlation functions

$$
\begin{aligned}
& \left\langle\varrho_{a}(n) \varrho_{a}\left(n^{\prime}\right)\right\rangle=\left\langle\varrho_{a}^{2}(n)\right\rangle K_{a}\left(n-n^{\prime}\right), \\
& \left\langle\varrho_{b}(n) \varrho_{b}\left(n^{\prime}\right)\right\rangle=\left\langle\varrho_{b}^{2}(n)\right\rangle K_{b}\left(n-n^{\prime}\right), \\
& \left\langle\varrho_{a}(n) \varrho_{b}\left(n^{\prime}\right)\right\rangle=\left\langle\varrho_{a}(n) \varrho_{b}(n)\right\rangle K_{a b}\left(n-n^{\prime}\right) .
\end{aligned}
$$




\section{Main result - Lyapunov exponent}

$$
\begin{aligned}
\lambda= & \frac{\varpi^{2}}{8 \sin ^{2} \gamma}\left[\sigma_{a}^{2} \mathcal{K}_{a}(2 \gamma) \sin ^{2} \varphi_{b}+\sigma_{b}^{2} \mathcal{K}_{b}(2 \gamma) \sin ^{2} \varphi_{a}\right. \\
& \left.-2 \sigma_{a b}^{2} \mathcal{K}_{a b}(2 \gamma) \sin \varphi_{a} \sin \varphi_{b} \cos \gamma\right],
\end{aligned}
$$

where $\sigma_{a, b}^{2}=k_{a, b}^{2}\left\langle\varrho_{a, b}^{2}(n)\right\rangle, \sigma_{a b}^{2}=k_{a} k_{b}\left\langle\varrho_{a}(n) \varrho_{b}(n)\right\rangle$, and $\sigma=Z_{a} / Z_{b}-Z_{b} / Z_{a}$ is the mismatching factor.

$\cos \gamma=\cos \varphi_{a} \cos \varphi_{b}-\frac{1}{2}\left(\frac{Z_{b}}{Z_{a}}+\frac{Z_{a}}{Z_{b}}\right) \sin \varphi_{a} \sin \varphi_{b}$ 


\section{Conventional Photonic Layered Media}

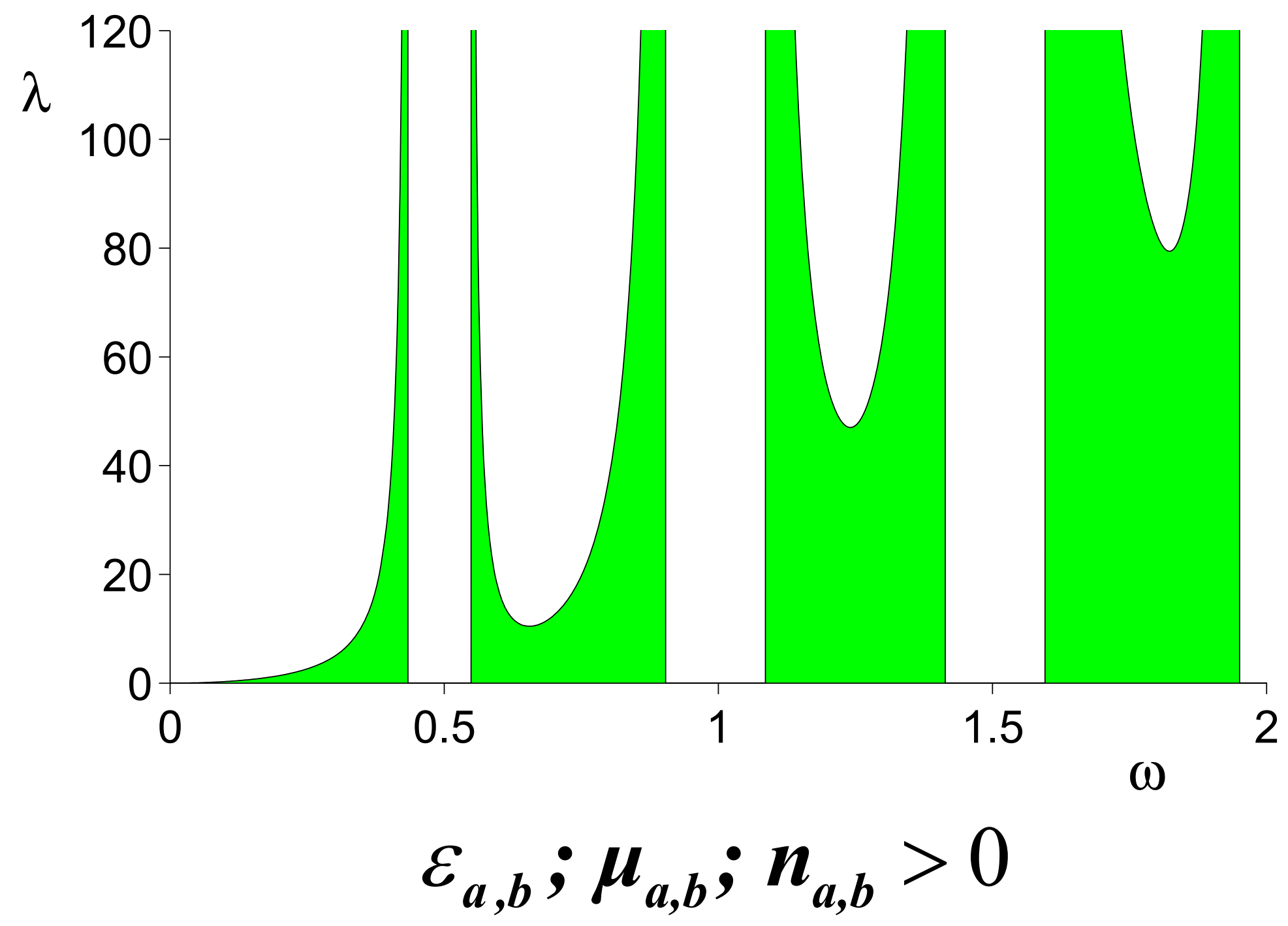




\section{Metamaterials}

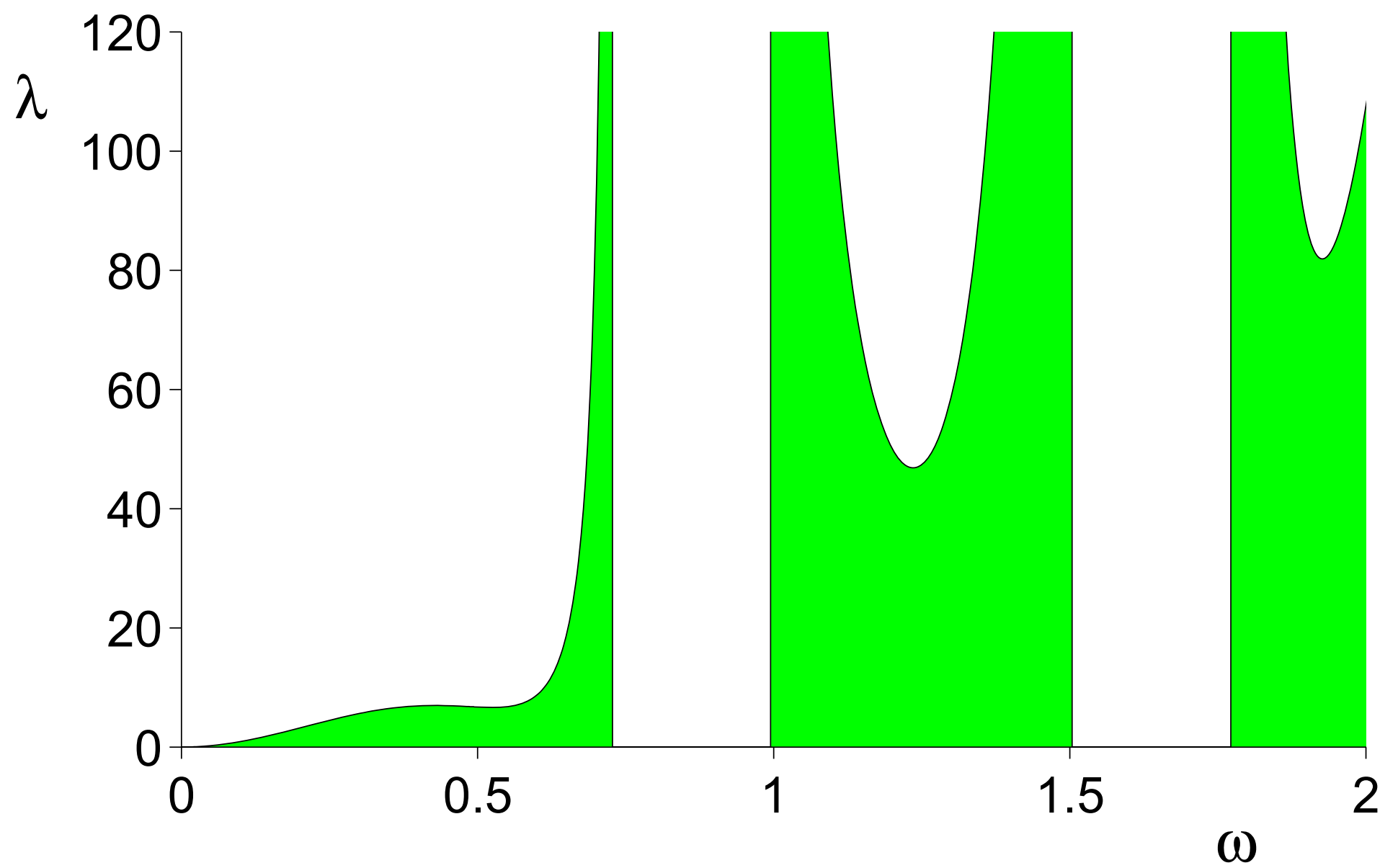

$$
\varepsilon_{a}, \boldsymbol{\mu}_{a}, \boldsymbol{n}_{a}>0 ; \quad \varepsilon_{b}, \boldsymbol{\mu}_{b}, \boldsymbol{n}_{b}<0
$$




\section{Electrons}

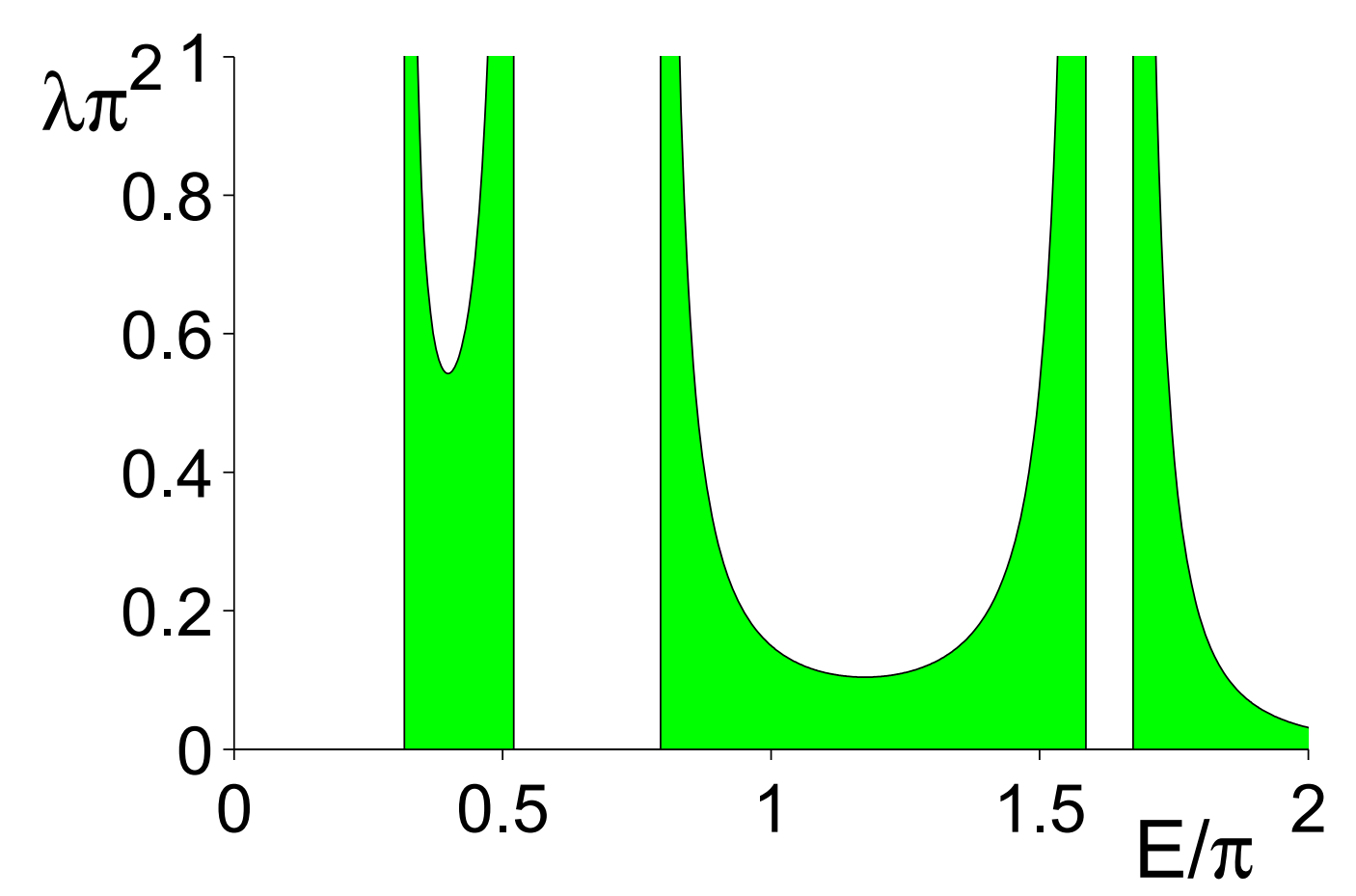

Cargese, July 12, 2010 


\section{Quarter stack layered medium $\quad \boldsymbol{n}_{\boldsymbol{a}} \boldsymbol{a}=\boldsymbol{n}_{\boldsymbol{b}} \boldsymbol{b}$}
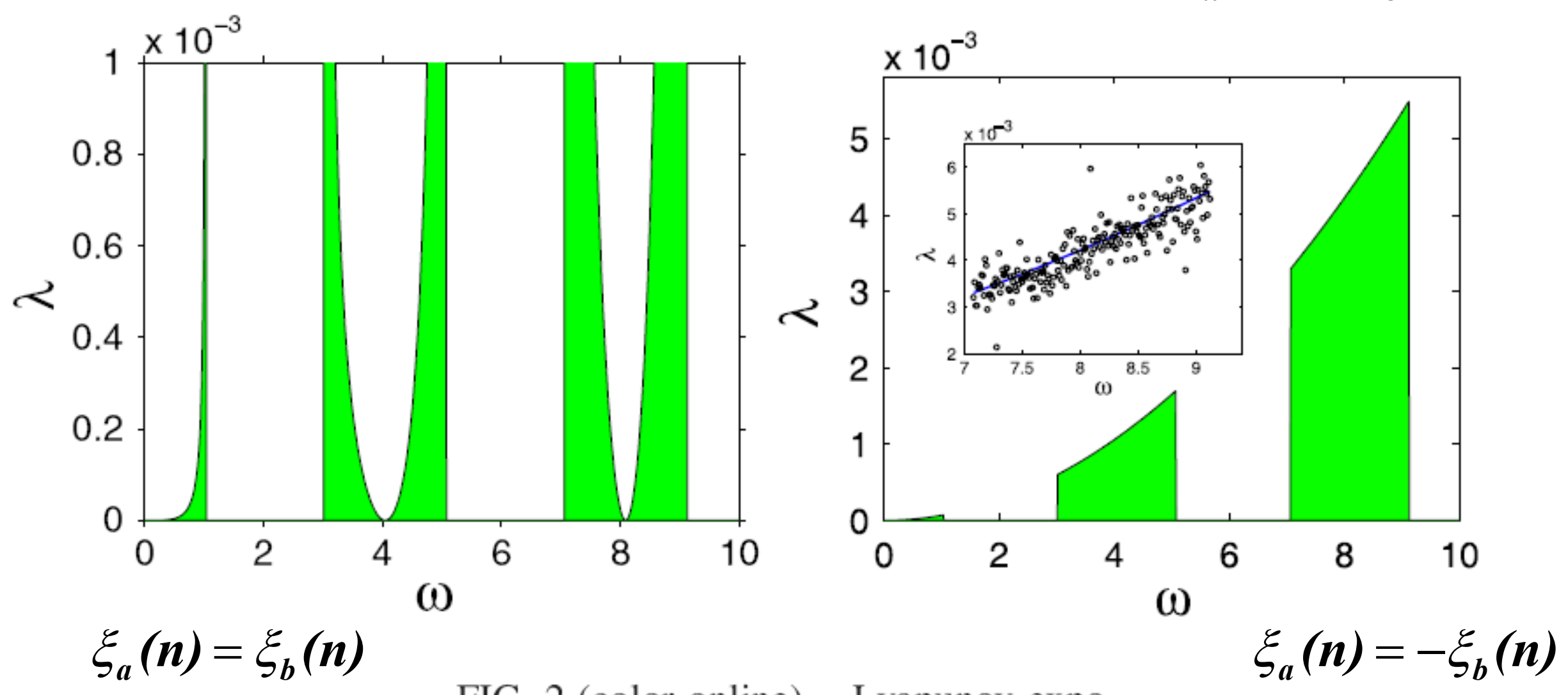

$$
\xi_{a}(n)=\xi_{b}(n)
$$

FIG. 2 (color online). Lyapunov expo-

$$
\xi_{a}(n)=-\xi_{b}(n)
$$
nent versus frequency for the quarter stack layered medium, for the plus correlations (left) and minus correlations (right); see Eqs. (8) and (10). Here $n_{a}=$ $3.47 \quad$ (silicon), $\quad d=1 \mu \mathrm{m}, \quad a=$ $0.224 \mu \mathrm{m},\left\langle\varrho_{a}^{2}(n)\right\rangle=3.75 \times 10^{-5} \mu \mathrm{m}^{2}$, $c=1.0$, and $\mathcal{K}_{a}=1$. 


\section{"Minus-correlations" $\quad \xi_{a}(n)=-\xi_{b}(n)$}

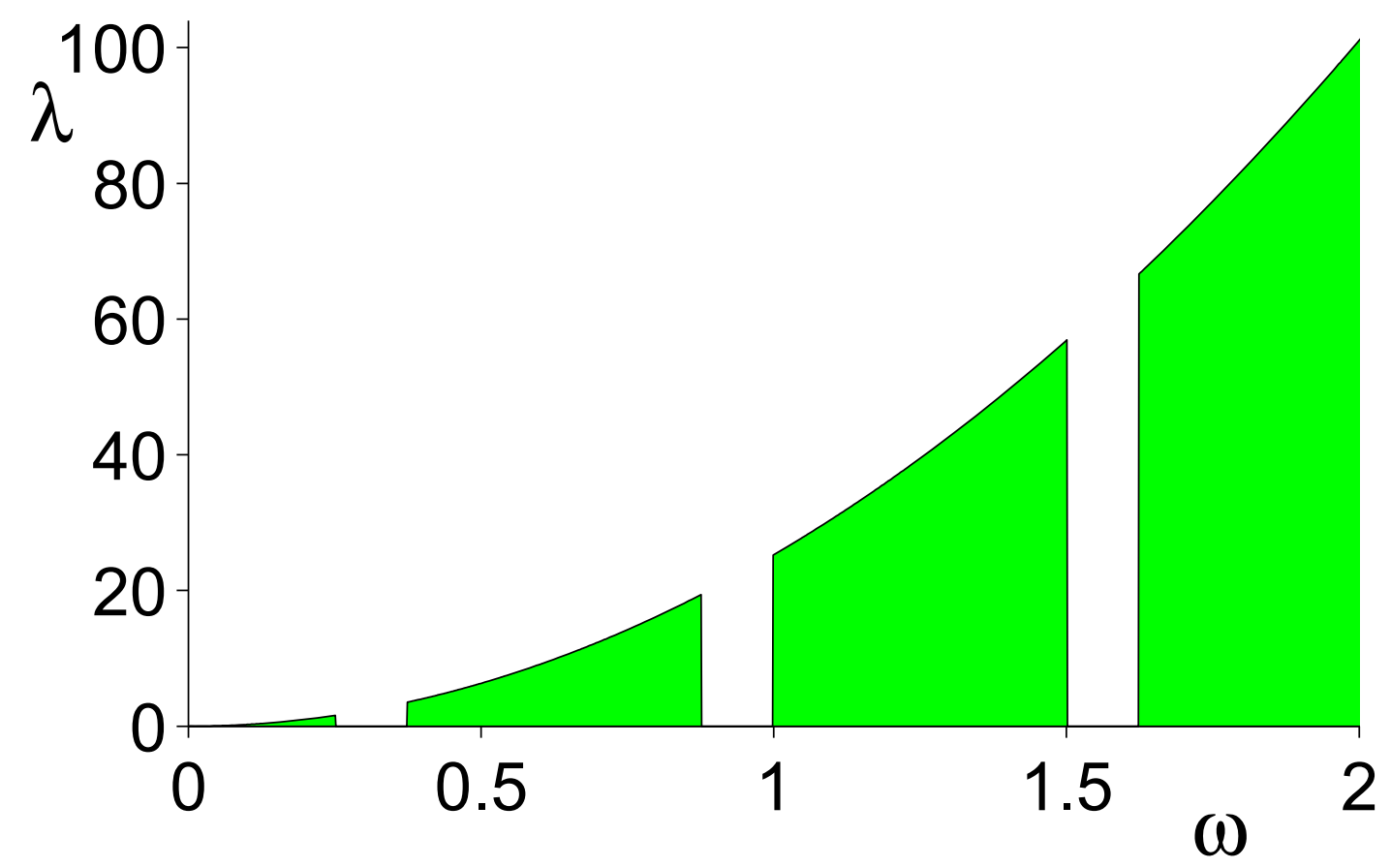




\title{
Anderson Localization in Bi-layer Array with Compositional Disorder: Conventional Photonic Crystals versus Metamaterials
}

\author{
F. M. Izrailev ${ }^{\mathrm{c}}$, N. M. Makarov ${ }^{*, b}$, E. J. Torres-Herrera ${ }^{\mathrm{c}}$ \\ ${ }^{a}$ Instituto de Física, Universidad Autónoma de Puebla, Apartado Postal J-48, Puebla 72570, México \\ ${ }^{b}$ Instituto de Ciencias, Universidad Autónoma de Puebla, Privada 17 Norte No. 3417, Col. San Miguel Hueyotlipan, Puebla 72050, México \\ ${ }^{c}$ Instituto de Física, Universidad Autónoma de Puebla, Apartado Postal J48, Puebla 72570, México
}

\begin{abstract}
The expression for the localization length has been derived for one-dimensional bi-layered structures with a weak random perturbation of the refractive index. Main attention is paid to the comparison between conventional materials and those consisting of mixed right-hand and left-hand materials. It is shown that the localization length has the same form for both cases.
\end{abstract}

Cargese, July 12, 2010 


\title{
One-dimensional Anderson localization in certain correlated random potentials
}

\author{
P. Lugan, A. Aspect, and L. Sanchez-Palencia \\ Laboratoire Charles Fabry de l'Institut d'Optique, CNRS and Univ. Paris-Sud, Campus Polytechnique, RD 128, \\ F-91127 Palaiseau Cedex, France
}

\author{
D. Delande \\ Laboratoire Kastler-Brossel, UPMC, ENS, CNRS, 4 Place Jussieu, F-75005 Paris, France \\ B. Grémaud \\ Laboratoire Kastler-Brossel, UPMC, ENS, CNRS, 4 Place Jussieu, F-75005 Paris, France
}

and Centre for Quantum Technologies, National University of Singapore, 3 Science Drive 2, Singapore 117543, Singapore

\section{A. Müller}

Laboratoire Kastler-Brossel, UPMC, ENS, CNRS, 4 Place Jussieu, F-75005 Paris, France and Physikalisches Institut, Universität Bayreuth, D-95440 Bayreuth, Germany

\author{
C. Miniatura \\ Centre for Quantum Technologies, National University of Singapore, 3 Science Drive 2, Singapore 117543, Singapore \\ and Institut Non Linéaire de Nice, UNS, CNRS, 1361 Route des Lucioles, F-06560 Valbonne, France \\ (Received 3 February 2009; revised manuscript received 4 June 2009; published 11 August 2009)
}

\begin{abstract}
We study Anderson localization of ultracold atoms in weak one-dimensional speckle potentials using perturbation theory beyond Born approximation. We show the existence of a series of sharp crossovers (effective mobility edges) between energy regions where localization lengths differ by orders of magnitude. We also point out that the correction to the Born term explicitly depends on the sign of the potential. Our results are in agreement with numerical calculations in a regime relevant for experiments. Finally, we analyze our findings in the light of a diagrammatic approach.
\end{abstract}




\title{
Inhomogeneous DNA: Conducting exons and insulating introns
}

\author{
A. A. Krokhin, ${ }^{1, *}$ V. M. K. Bagci, ${ }^{1}$ F. M. Izrailev, ${ }^{2}$ O. V. Usatenko, ${ }^{3}$ and V. A. Yampol'skii ${ }^{3}$
}

Parts of DNA sequences known as exons and introns play very different roles in coding and storage of genetic information. Here we show that their conducting properties are also very different. Taking into account long-range correlations among four basic nucleotides that form double-stranded DNA sequence, we calculate electron localization length for exon and intron regions. Analyzing different DNA molecules, we obtain that the exons have narrow bands of extended states, unlike the introns where all the states are well localized. The band of extended states is due to a specific form of the binary correlation function of the sequence of basic DNA nucleotides. 